PSYCHOMETRIKA-VOL. 45, NO. I

MARCH, 1980

NOTES AND COMMENTS

\title{
A NEW SOLUTION TO THE PROBLEM OF FINDING ALL NUMERICAL SOLUTIONS TO ORDERED METRIC STRUCTURES
}

\author{
Paul E. Lehner and Elliot Noma \\ THE UNIVERSITY OF MICHIGAN
}

\begin{abstract}
A new algorithm is used to test and describe the set of all possible solutions for any linear model of an empirical ordering derived from techniques such as additive conjoint measurement, unfolding theory, general Fechnerian scaling and ordinal multiple regression. The algorithm is computationally faster and numerically superior to previous algorithms.
\end{abstract}

Key words: ordered metric structures, convex cone, additive conjoint measurement, unfolding theory, Fechnerian scaling.

In 1975, McClelland and Coombs described an algorithm for constructing all numerical solutions to a set of homogeneous linear inequalities. The general purpose of their algorithm, called ORDMET, was to test and describe the set of all possible solutions for any linear model of an empirical ordering derived from such techniques as additive conjoint measurement, unfolding theory, general Fechnerian scaling, and ordinal multiple regression. Generally speaking, these empirical orderings generate linear inequalities which describe the ordering of differences, (i.e., ordered metric structures).

Briefly summarized, the problem ORDMET addresses is as follows. We are given a set of inequalities:

$$
\text { As } \geq 0
$$

where $\mathbf{A}$ is a matrix of integer coefficients and $\mathbf{s}$ is the vector of variables. We want to find all positive solutions to (1), giving us the system of inequalities,

$$
\begin{gathered}
\text { As } \geq 0 \\
\mathbf{s} \geq 0 .
\end{gathered}
$$

By well-known results from linear algebra [see for example Murty, 1976], the inequalities in (2) define a convex polyhedral cone. That is, there exists a matrix $\mathbf{V}=$ $\left[\mathbf{v}_{1} \vdots \mathbf{v}_{2} \vdots \cdots \vdots \mathbf{v}_{m}\right]$, such that,

(i) no column in $V$ is a positive linear combination of the others, and

(ii) $\mathbf{s}$ satisfies (2) if and only if there exists a set of positive scalars, $\left\{r_{1}, r_{2}, \cdots, r_{m}\right\}$, such that,

$$
\mathbf{s}=\sum_{i=1}^{m} r_{i} \mathbf{v}_{i}
$$

From (ii) above, it is easy to see that a vector of stimulus scale values, s, satisfies (1) if and only if there exists a vector of positive scalars $\mathbf{r}$, and a constant vector $\mathbf{b}$, such that,

$$
\mathbf{S}=\mathbf{V r}+\mathbf{b} .
$$

This research was supported in part by NIGMS grant GM-0123I to the University of Michigan. Authors' names are in alphabetic order.

Requests for reprints should be sent to Paul E. Lehner, Department of Psychology, 580 Union Drive, University of Michigan, Ann Arbor, MI, 48109. 
The columns of $\mathbf{V}$ are called extreme points in the convex polyhedral cone defined by (2). Each extreme point is unique up to multiplication by a positive scalar. The purpose of ORDMET is to derive this $V$ matrix of extreme points.

Unfortunately, the ORDMET procedure, and its predecessors, have some problems. Goode's version [Goode, 1964; see also Coombs, 1964, p. 97-102] processed most inequalities, but often generated unresolved side constraints. Phillips [1971] developed an algorithm which eliminated these side constraints, but it generated a $\mathrm{V}$ matrix with many redundant columns. These columns were redundant because they could be expressed as positive linear combinations of other columns in the $\mathbf{V}$ matrix. McClelland and Coombs [1975] used Phillips' algorithm and then applied a method developed by Wetz and Witzgall [1966] to eliminate the redundant columns. However, the implementation of the Wetz and Witzgall algorithm is, by itself, a numerical problem. In particular, three of the four tests for redundancy [Step 6, in McClelland \& Coombs, 1975, p. 274] require a decision as to whether column items are zero or non-zero. Because of computational roundoff errors, this decision cannot be made directly. Rather, tolerance levels must be set to determine how small a number must be before it is considered to be a zero by the column reduction algorithm. Therefore, in order to guarantee that any particular implementation of ORDMET produces accurate results, it must be shown that the system used to establish these tolerance levels is appropriate. Consequently, the ORDMET algorithm as it is described in McClelland and Coombs [1975] is incomplete.

Because of this difficulty, an alternative to the ORDMET algorithm is desired. As it turns out, an alternative already exists. This algorithm was developed independently by Motzkin, Raiffa, Thompson and Thrall [1953], and Chernikova [1965]. A description of the algorithm, as it is implemented in the program ORDMET2*, is given below.

Chernikova's algorithm has several advantages over ORDMET. First, Chernikova's algorithm does not produce redundant columns. Therefore, column reduction is not needed. Second, the implementation of the algorithm uses only integer arithmetic. Therefore, there is no need to set tolerance levels to determine if a value is zero or not. Also, the implementation of Chernikova's algorithm requires considerably less CPU time and core storage than ORDMET. This is because there are fewer processing steps. In addition, the explosion of redundant $\mathbf{V}$ columns that sometimes results from the Phillips procedure does not occur.

\section{Algorithm}

The following description of the algorithm was adapted from the description of Chernikova's algorithm found in Appendix D of Mattheiss and Rubin [Note 1] (This paper was brought to our attention by Professor Katta Murty.).

To find all extreme points in the convex cone defined by $\{s:$ As $\geq 0, s \geq 0$, where $A$ is an $m$ by $n$ matrix of integers we process the matrix $[A]$, where $I$ is an $n$ by $n$ identity matrix. A series of transformations of this matrix will generate the $V$-matrix. At any stage in the process let the transformed matrix be denoted by $\mathbf{Y}=\left[\begin{array}{l}\mathrm{U} \\ \mathbf{L}\end{array}\right]$. The matrices $\mathbf{U}$ and $\mathbf{L}$ will always have $m$ and $n$ rows respectively, but the number of columns in $Y$ will change as the algorithm proceeds. Note that initially $\mathbf{U}=\mathbf{A}$ and $\mathbf{L}=\mathbf{I}$.

Each iteration of the algorithm will construct a new $\mathbf{Y}$ from the present $\mathbf{Y}$. Initially, $\mathbf{Y}$ $=\left[\begin{array}{l}\mathrm{A} \\ ]\end{array}\right]$. The algorithm proceeds as follows.

Step 0: (check if finished)

(1) If any row of $U$ has only zero or negative components, then $s=0$ is the only solution, and we are done. Otherwise, go to Step O(2).

* This program is available from Professor James Lingoes, room 1005, North University Building, Computer Center, University of Michigan, Ann Arbor, Michigan 48109. 
(2) If all the elements of $U$ are nonnegative, then the matrix $L$ is the $V$ matrix, and we are done. Otherwise, go to Step 1.

Step 1: (choose row to be processed)

Choose the first row of $\mathbf{U}$, say row $r$, with at least one strictly negative element. Go to Step 2(1).

Step 2: (construct new $\mathbf{Y}$ )

(1) Install as columns in the new $\mathbf{Y}$ all columns of the present $\mathbf{Y}$ for which $y_{r j} \geq 0$. Go to Step 2(2).

(2) Let $I_{0}$ be the index set of all nonnegative rows for the current $\mathbf{Y}$. Find all pairs $(s, t)$, such that $y_{r s} \cdot y_{r}<0$ and $s<t$. Call this set $S$. With the first element of $S$, go to (a).

(a) Find all $i \in I_{0}$, such that $y_{i s}=0$ and $y_{i t}=0$. Call this set $I_{2}(s, t)$. Go to (b).

(b) If $I_{1}(s, t)$ is empty, go to (e). Otherwise, go to (c).

(c) If $I_{1}(s, t)$ is not empty, check if there is a $u$, not equal to either $s$ or $t$, such that $y_{i u}=0$ for all $i \in I_{1}(s, t)$. If such a $u$ exists, go to (e). Otherwise, go to (d).

(d) Add a new column to the new $\mathbf{Y}$ which is equal to,

$$
\left[\frac{y_{i t}}{\operatorname{GCD}\left(y_{i s}, y_{i t}\right)} \mathbf{y}_{s}\right]+\left[\frac{y_{i s}}{\operatorname{GCD}\left(y_{i s}, y_{i t}\right)} \mathbf{y}_{\cdot}\right]
$$

where $\operatorname{GCD}(a, b)$ is the greatest common denominator of the positive integers $a$ and $b$. Go to (e).

(e) Go to (a) with the next $(s, t)$ pair in $S$. If all the elements of $S$ have been examined, go to Step 3.

Step 3: (set up next iteration)

At this point, the new $Y$ has been completed. Set the present $Y$ to be the new $Y$, and go to Step $0(1)$ to construct the next new $Y$.

\section{REFERENCE NOTE}

1. Mattheiss, T. H. \& Rubin, D. S. A survey and comparison of methods for finding all vertices of convex polyhedral sets (Tech. Rep. No. 77-14). Chapel Hill: Curriculum in Operations Research, University of North Carolina at Chapel Hill, November 1977.

\section{REFERENCES}

Chernikova, N. V. Algorithm for finding a general formula for the nonnegative solutions of a system of linear inequalities. U.S.S.R. Computational Mathematics and Mathematical Physics, 1965, 5, 228-233.

Coombs, C. H. A theory of data. New York: Wiley, 1964.

Goode, F. M. An algorithm for the additive conjoint measurement of finite data matrices. American Psychologist, 1964, 19, 579.

McClelland, G. H. \& Coombs, C. H. ORDMET, A general algorithm for constructing all numerical solutions to ordered metric structures. Psychometrika, 1975, 40, 269-290.

Motzkin, T. S., Raiffa, H., Thompson, G. L., \& Thrall, R. M., The double description method. In H. W. Kuhn \& A. W. Tucker (eds.), Contributions to the Theory of Games, II. Annals of Mathematics Study, No. 28, Princeton, N.J.: Princeton University Press, 1953.

Murty, K. G. Linear and combinatorial programming, New York: Wiley, 1976.

Phillips, J. P. N. A note on the representation of ordered metric scaling. British Journal of Mathematical and Statistical Psychology, 1971, 24, 239-250.

Wetz, R. J. B. \& Witzgall, C. Algorithms for frames and linearity of cones. Journal of Research of the National Bureau of Standards, 1967, 71B, 1-7. 